\title{
Effects of Power Line Communication on Radio Communication Equipment
}

\author{
Ganiyu Adedayo Ajenikoko Babajide Akinjobi \\ Department of Electronic and Electrical Engineering, Ladoke Akintola University of Technology, P.M.B, 4000, \\ Ogbomoso, Nigeria
}

\begin{abstract}
Radio communication is a wireless transfer of information via power cable by Power Line Communication (PLC) and its dissemination through radio communication equipment.. However, this information causes signal interference, noise and distortion with the power line communication. This work analyzed the effect of PLC on radio communication equipment by interconnecting two high-speed PLC modems communicating with a data rate of up to $250 \mathrm{Mbit} / \mathrm{s}$ using telephone distribution wiring for radio spectrum to reduce the interference radiation that emanates from a power line. The measurements were made using a reference antenna at a distance of $3 \mathrm{~m}$ from the power line. The peak field-strength values were measured and recorded in the $30-350 \mathrm{MHz}$ frequency range in horizontal and vertical polarization. The result shows that interference radiation occurring at frequencies up to $305 \mathrm{MHz}$, includes the FM band as well as the Digital Audio Broadcasting (DAB) band. It is expected that as the data rate of high speed modems increases, a corresponding increase in the interference radiation occurs at frequencies above $300 \mathrm{MHz}$. To this regard, administrations should take all practicable and necessary steps to ensure that the operation of power and telecommunication distribution networks do not cause harmful interference to a radio communication service.
\end{abstract}

Keywords: PLC, Radio Communication, Interference, Noise, Distortion, Communication Equipment, Field Strength, DAB

DOI: $10.7176 / \mathrm{JNSR} / 9-16-04$

Publication date: August $31^{\text {st }} 2019$

\section{INTRODUCTION}

Power line communication (PLC) is one of the technologies that have proved useful for control applications. It is widely use in home automation, automotive and internet access applications. Power line communication systems operate by adding a modulated carrier signal to the wiring system [1]. Since power distribution system was originally intended for transmission of AC power at typical frequencies of 50 or $60 \mathrm{~Hz}$, power wire circuits have only a limited ability to carry higher frequencies. The propagation problem is a limiting factor for each type of power line communication. Many nations regulate unshielded wired emissions as if they were radio transmitters. These jurisdictions usually require unlicensed use to be below $500 \mathrm{KHz}$ or in unlicensed radio bands $[2,3,4]$.

A radio communication consists basically of a transmitter and a receiver. The transmitter contains an oscillator which generates Radio Frequency (RF) energy in the form of alternating current (AC) [2]. A transmission line or cable feeds the RF to the antenna. The antenna converts the AC into electromagnetic energy which is radiated into space. The role of the radio communication sector is to ensure the rational, equitable, efficient and economical use of the radio-frequency spectrum by all radio communication services, including satellite services, and carry out studies without limit of frequency range [1,5].

PLC also known as a carrier current system is used for transmitting radio programs over power lines when operates in the AM radio band. Normally, in single-channel radio operations, the receiver uses the same antenna as the transmitter to receive electromagnetic energy [3]. The antenna converts the received electromagnetic energy into RF alternating current. The RF is fed to the receiver by a transmission line or cable. In the receiver, the RF is converted to audio frequencies (AF). The audio frequencies are then changed into sound waves by a headset or loud speaker $[5,6]$.

In addition, utility companies use special coupling capacitors to connect radio transmitters to the powerfrequency AC conductors. Frequencies used are in the range of 24 to $500 \mathrm{kHz}$, with transmitter power levels up to hundreds of watts. These signals may be impressed on one conductor, on two conductors or on all three conductors of a high-voltage AC transmission line [1,7]. Several PLC channels may be coupled onto one High Voltage (HV) line. Filtering devices are applied at substations to prevent the carrier frequency current from being bypassed through the station apparatus and to ensure that distant faults do not affect the isolated segments of the PLC system. These circuits are used for control of switchgear, and for protection of transmission lines. It is on this above direction that this work intends to analyze the effect of power line communication on radio equipment so as to reduce the interference radiation that emanates from a power line when used for transmitting radio programs $[3,7,8]$. 


\section{A. Power Line Communication}

Power line communication (PLC) is a communication technology that enables the sending data over existing power cables. Power line carrier communication has recently become a popular technology for home automation and networking. It is because power line is a relatively cheaper and more robust communication channel used throughout the world except wireless channel [4]. It is more commonly used than any other communication channel. A power line modem is an all-in-one device which consists of an encoder, a decoder, a modulator, and a demodulator. As the current bearing AC mains power line is used as a transmission medium, additional coupling circuits are required in power line modems for better protection, isolation and impedance matching. Power line modems can be used in various applications $[1,5,9]$.

PLC is like any other communication technology whereby a sender modulates the data to be sent, injects it onto medium, and the receiver de-modulates the data to read it. The major difference is that PLC does not need extra cabling, it re-uses existing wiring. Considering the pervasiveness of power lines, this means with PLC, virtually all line-powered devices can be controlled or monitored [3, 8, 9].

PLC are broadly viewed as narrowband PLC and broadband PLC. Narrow band PLC works at lower frequencies $(3-500 \mathrm{kHz}$ ), lower data rates (up to $100 \mathrm{~s}$ of $\mathrm{kbps}$ ) and has longer range (up to several kilometers) which can be extended using repeaters. Broadband PLC works at higher frequencies $(1.8-250 \mathrm{MHz})$ high data rates (up to 100s of Mbps) and is used in shorter range applications. Broadband PLC, in contrast, has mainly found acceptance as a last mile solution for internet distribution and home networking [1]. With its high data rates and no additional wiring, broadband PLC is seen as an exciting and effective technology for multimedia distribution within homes [9].

Narrowband line communication systems sometimes operate within the frequency range from three kilocycle to five hundred kilocycle and broadband line communication system sometimes operates within the frequency range from one megacycle to three hundred megacycle. The block diagram of PLC is shown in Figure $1[6]$.

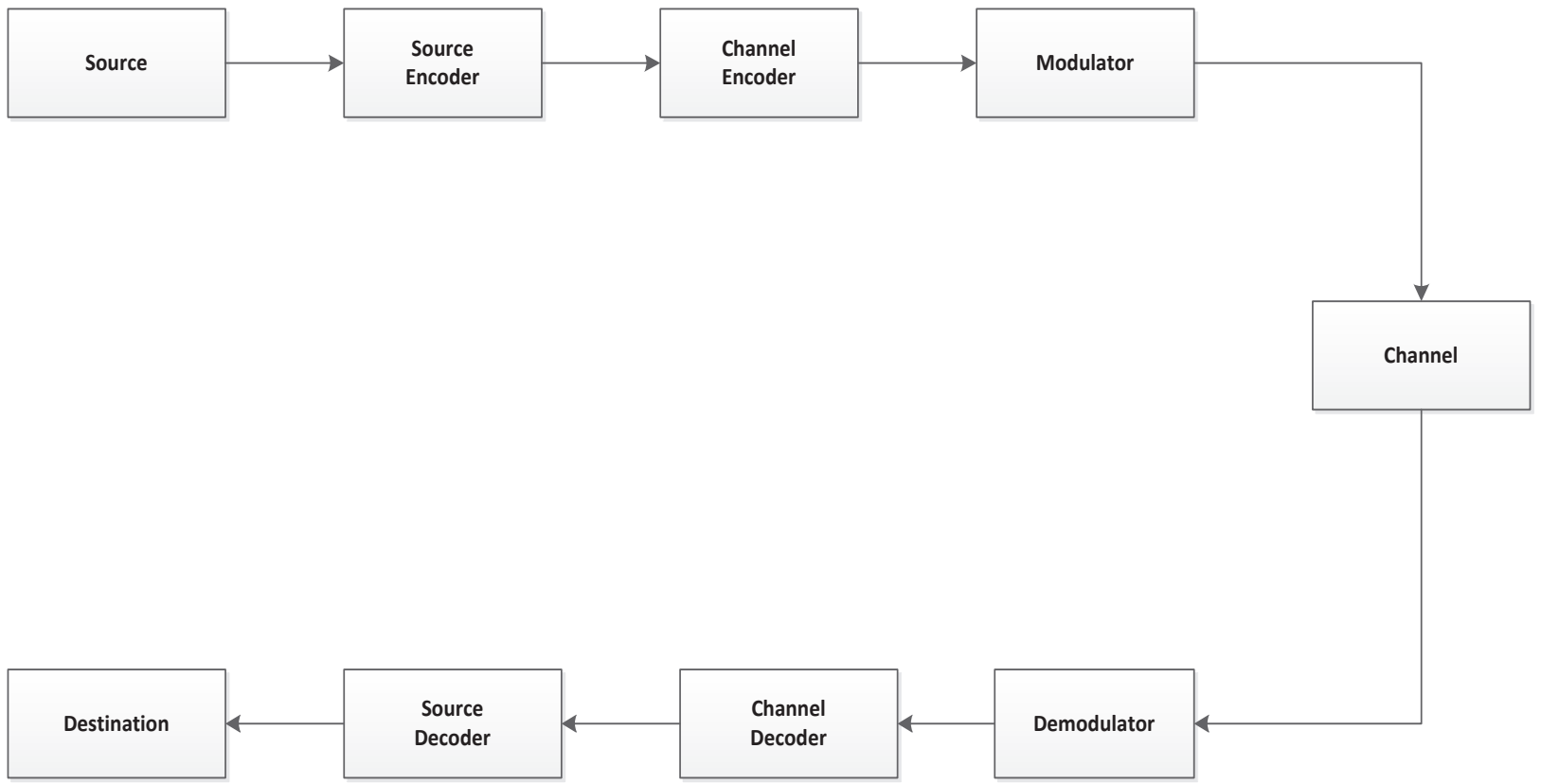

Figure 1: Block Diagram of Power line communication

\section{B. Radio Communication}

Radio is the technology of signaling or communication using radio waves. Radio waves are electromagnetic waves of frequency between 30 hertz $(\mathrm{Hz})$ and 300 gigahertz $(\mathrm{GHz})$ [7]. They are generated by an electronic device called a transmitter connected to an antenna which radiates the waves, and received by a radio receiver connected to another antenna. In radio communication, radar, radio navigation, remote control, remote sensing, cell phones, two-way radios, wireless networking and satellite communications are used to carry information across space from a transmitter to a receiver, by modulating the radio signal in the transmitter [10, 11].

In radio communication systems, information is carried across space using radio waves. At the sending end, the information is converted by transducer to a time-varying electrical signal called the modulation signal [6]. The modulation signal applied to a radio transmitter may be an audio signal representing sound from a microphone, a video signal representing moving images from a video camera, or a digital signal consisting of a 
sequence of bits representing binary data from a computer [10]. In the transmitter, an electronic oscillator generates an alternating current oscillating at a radio frequency, called the carrier wave because it carries the information through the air. The information signal is used to modulate the carrier, varying some aspect of the carrier wave, impressing the information on the carrier $[9,11,12]$.

Different radio communication systems used different modulation methods such as $[4,9,12]$ :

1. Amplitude Modulation (AM): In an AM transmitter, the amplitude of the radio carrier wave is varied by the modulation signal..

2. Frequency Modulation (FM): In an FM transmitter, the frequency of the radio carrier wave is varied by the modulation signal.

3. Frequency Shift Keying (FSK): Used in wireless digital devices to transmit digital signals, the frequency of the carrier wave is shifted periodically between two frequencies that represent the two binary digits, 0 and 1, to transmit a sequence of bits.

4. Orthogonal Frequency Division Multiplexing (OFDM): OFDM has higher spectral efficiency and more resistance to fading than AM or FM. Multiple radio carrier waves closely spaced in frequency are transmitted within the radio channel, with each carrier modulated with bits from the incoming bit stream so that multiple bits are being sent simultaneously, in parallel. At the receiver, the carriers are demodulated and the bits are combined in the proper order into one bit stream.

\section{Radio Communications Issues}

The main radio communication issue raised by the widespread use of broadband power line communications systems is the risk of interference to radio communication services caused by generation of electromagnetic emissions from the power lines $[10,13]$. Open wire aerial power lines in particular, freely radiate high frequency signals being passed along their length. The high frequency signals in this case are typically generated by faults in the wired network such as arcing switch gear, coronal discharge and discharges across dirty insulators. Similar experiences across the world have led many to believe that the deliberate placement of high frequency signals on power lines by broadband power line communication systems lead to large scale interference problems for radio communication services particularly in the High Frequency (HF) spectrum (3 to $30 \mathrm{MHz})[11,14,15]$.

The broadband power line communication systems consist of two or more terminal devices connected via the AC power supply network [14]. Broadband power line communication systems fall under the definition of a "transmitter" because it gives rise to radio emissions even though these emissions are not intentional. However, broadband power line communication, systems are not radio communication transmitters as the intended path of communications between the devices is through the wiring of the AC supply network and not by radio waves. The electromagnetic radiation from the wiring is an unintentional consequence of the communication along the wiring $[15,16,17]$.

For practical purposes, a communication system is considered to be wired or wireless (e.g., conventional telephone, radio communications). A wired system is technically known as a hard-line system and can be thought of as a localized, private telephone system that uses wires to operate over a limited area [16, 17]. A wireless system uses radio frequencies to connect users and is capable of operating over a much larger geographical area than a hard-line (wired) system. The major advantages of RF communication systems over hard-line communication systems are their ability to provide communications over large distances, through some obstacles, and to an almost unlimited number of users. The range of the signal is defined to be the distance between the transmitter and the receiver at which the amplitude of the signal received by the receiver is less than the amplitude of the background noise [5, 17, 18].

\section{Radio Communication Equipment}

Radio communication equipment is equipment that facilitates or helps in the transmission and reception of signals, messages, information and data wirelessly [18]. This includes the equipment used in the medical firms, radio broadcasting, TV broadcasting, satellite communication, mobile cellular telephony, wireless LAN, multimedia communication and mobile internet [13]. However, the major components of a radio communication equipment PLC channel includes; transmitters, receivers, line tuners, filters, line traps, couplers, injectors and extractors. The problem associated with the PLC channel is the requirement to put the carrier signal onto the high voltage line without damaging the carrier equipment. Once the signal is on the power line it must be directed in the proper direction in order for it to be received at the remote line terminal. Some of these components are $[1,5,14,16,17]$ :

1. Transmitters and Receivers: The simplest radio transmitter consists of a power supply and an oscillator. The carrier transmitters and receivers are usually mounted in a rack or cabinet in the control house, and the line tuner is out in the switchyard. This then means there is a large distance between the equipment and the tuner, and the connection between the two is made using a coaxial cable. The coaxial cable provides shielding so that noise cannot get into the cable and cause interference. The coaxial cable 
is connected to the line tuner which must be mounted at the base of the coupling capacitor.

2. Continuous Wave (CW) transmitter: A radio transmitter is used to generate RF energy which is radiated into space. The output of the oscillator is applied to a buffer stage to increase oscillator stability and to a power amplifier which produces greater output. A telegraph key is used to control the energy waves produced by the transmitter. When the key is closed, the transmitter produces its maximum output. When the key is opened, no output is produced.

3. Antennas: After an RF signal has been generated and amplified in the transmitter, it is radiated into space by an antenna. At the distant station, a receiving antenna is used to receive the signal from space. An antenna consists of wires or rods designed for use with either a radio transmitter or a radio receiver.

4. Detector: Detection is a process of recovering intelligence from an RF signal, and the circuit in which it occurs is called a detector. The detector recovers the intelligence from the carrier and makes it available for direct use or for further amplification. In an FM receiver, the detector is usually called a discriminator.

5. Radio frequency amplifier: The RF amplifier normally uses tunable circuits to select the desired signal. It contains transistors, electron tubes, or integrated circuits (IC) to amplify the signal to a usable level.

6. Hybrids and Filters: Hybrid circuits enable the connection of two or more transmitters together on one coaxial cable without causing inter-modulation distortion due to the signal from one transmitter affecting the output stages of the other transmitter. Hybrids are also required between transmitters and receivers, depending on the application. The hybrid circuits can cause large losses in the carrier path and must be used appropriately.

7. Line Tuners: The purpose of the line tuner in conjunction with the coupling capacitor is to provide a low impedance path for the carrier energy to the transmission line and a high impedance path to the power frequency energy. The line tuner and coupling capacitor combination provides a low impedance path to the power line by forming a series resonant circuit tuned to the carrier frequency.

8. Line Traps: The line trap is usually a form of a parallel resonant circuit which is tuned to the carrier energy frequency. A parallel resonant circuit has high impedance at its tuned frequency, and it then causes most of the carrier energy to flow toward the remote line terminal. The coil of the line trap provides a low impedance path for the flow of the power frequency energy.

9. L/C Filters: L/C filters are used to combine two or more transmitters. The bandwidth response of the series resonant L/C filter is a function of the L: C ratio and the frequency to which it is tuned.

10. Coupling Capacitors: Coupling capacitor is a device which provides a low impedance path for the carrier energy to the high voltage line, and at the same time blocks the power frequency current by being a high impedance path at those frequencies. It can only perform its function of dropping line voltage across its capacitance if the low voltage end is at ground potential.

\section{MATERIALS AND METHOD.}

This work covers the use of radio spectrum and associated protection requirements of radio communication services to analyze the effect of Power Line Communications (PLC) on radio communication equipment. In this work two high-speed PLC modems communicating with a data rate of up to $250 \mathrm{Mbit} / \mathrm{s}$ were interconnected to reduce the interference radiation that emanates from a power line when used as radio communication. The measurements were made using a reference antenna at a distance of $3 \mathrm{~m}$ from the power line. The peak fieldstrength values were measured and recorded in the 30 to $350 \mathrm{MHz}$ frequency range in horizontal and vertical polarization.

The assumptions made for PLC modem are the following:

i. The total interference at the receiver from all radiations and emissions without a corresponding frequency allocation in the radio regulations should not exceed $1 \%$ of the total receiving system noise power.

ii. The total interference at the receiver arising from all sources of radio-frequency emissions from radio communication services with a corresponding co-primary frequency allocation should not exceed $10 \%$ of the total receiving system noise power.

iii. In order to limit receiver sensitivity deterioration within $0.05 \mathrm{~dB}$, the requirement for protecting the broadcasting service should be $20 \mathrm{~dB}$ lower than the equivalent field strength of the environmental noise in bandwidth.

The protection requirement can be expressed in terms of a maximum field strength density of $\mathrm{dB}(\mu \mathrm{V} / \mathrm{m} / \mathrm{MHz})$ with $b=1 \mathrm{MHz}$. The protection requirement is expressed by:

$$
\text { maximum field strength density }=g+h \log f \quad d b(\mu \mathrm{V} / \mathrm{m} / \mathrm{MHz})
$$




$$
\begin{aligned}
& g=c-55.5 \\
& h=20-d \\
& g \text { and } h \text { are radiated emissions classes, } f \text { is the frequency, } c \text { is constant and } d \text { is the distance. }
\end{aligned}
$$

\section{RESULTS AND DISCUSSION}

The analysis of results for the field strength of signal radiation emanated from power line communication system for 30 to $350 \mathrm{MHz}$ frequency range in horizontal and vertical polarization is presented in Figure 2 and 3.

Figure 2 shows the relationship between field strength in $\mathrm{dB}(\mu \mathrm{V} / \mathrm{m})$ and frequency range of the radio spectrum for horizontal polarization. The peak values of the field-strength between 87-108 MHz range for FM signals and those between $220-270 \mathrm{MHz}$ range match the DAB signals. The measuring receiver's system noise is the smallest measurable field strength while the environmental noise in absence of the modems is below 150 MHz. This is due to radiation interference that occurs from the PLC modem. When the modems are switched on, the interference field strength in idle mode increased. In addition, during data transfer, the interference field strength also increased. However, above $145 \mathrm{MHz}$, the level of the interference of field strength caused by the modems improved above the environmental noise. This shows that the measured values are correct.

Figure 2 shows the relationship between the field strength in $\mathrm{dB}(\mu \mathrm{V} / \mathrm{m})$ and frequency range of the radio spectrum for vertical polarization. The result shows that FM signals at 85 to $125 \mathrm{MHz}$ and DAB signals at 225 $\mathrm{MHz}$ to $275 \mathrm{MHz}$ are higher field strength measurements values since they are transmitted at a vertical polarization. The peak values of the modems interference during data transfer and in idle mode are above the limit values in the entire frequency range. In addition, the results also show that the radiations from PLC are comparable to the field-strengths for reception. Effect of PLC reduced the noise acceptable threshold and audio quality can quickly degrade from noisy to unintelligible level with only a small variation.

Therefore, the spectral measurements on high-speed PLC modems show interference radiation occurring at frequencies up to $305 \mathrm{MHz}$, which includes the FM band and DAB band. The measurements also show that as the data rate of high speed modems increases, a corresponding increase in the interference radiation occurs at frequencies above $300 \mathrm{MHz}$.

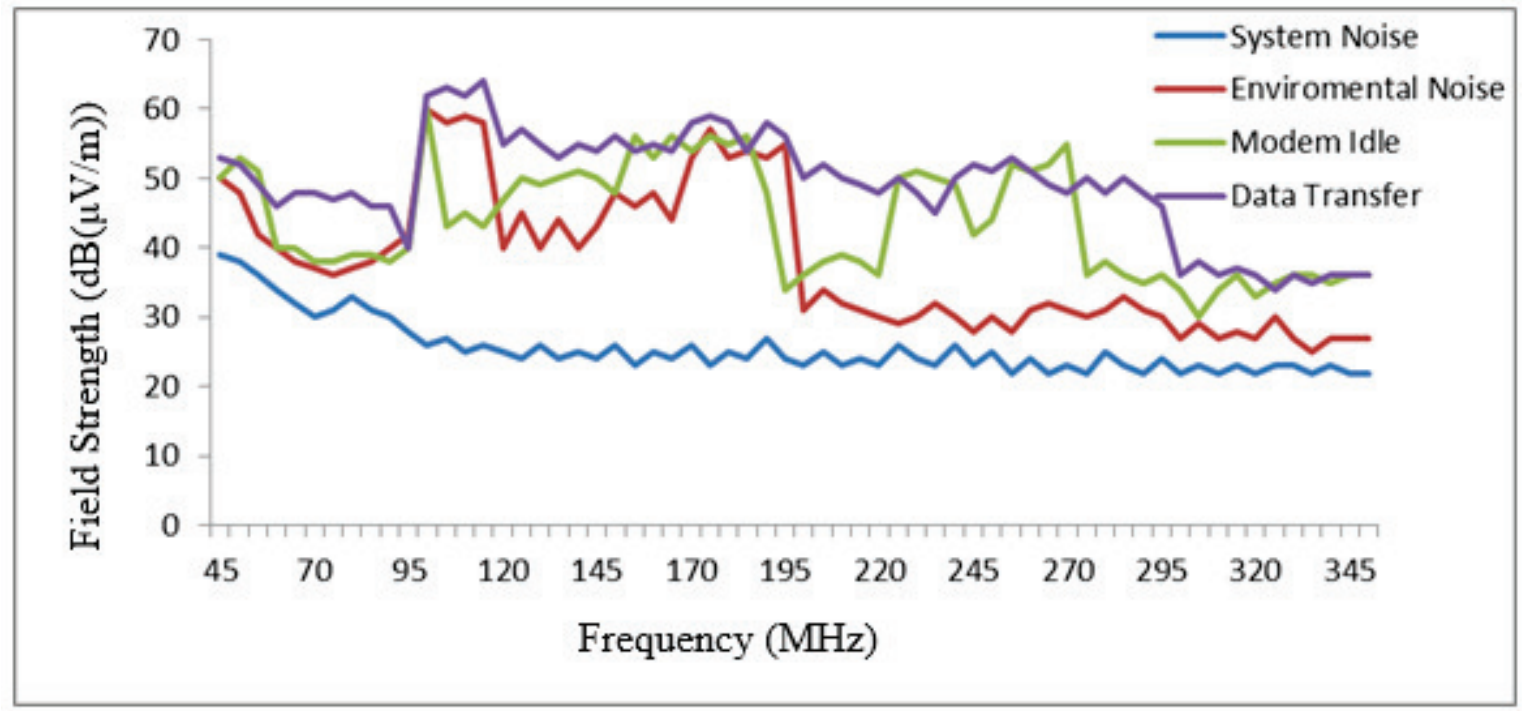

Figure 1: PLC Interference Field Strength with Horizontal Polarization 


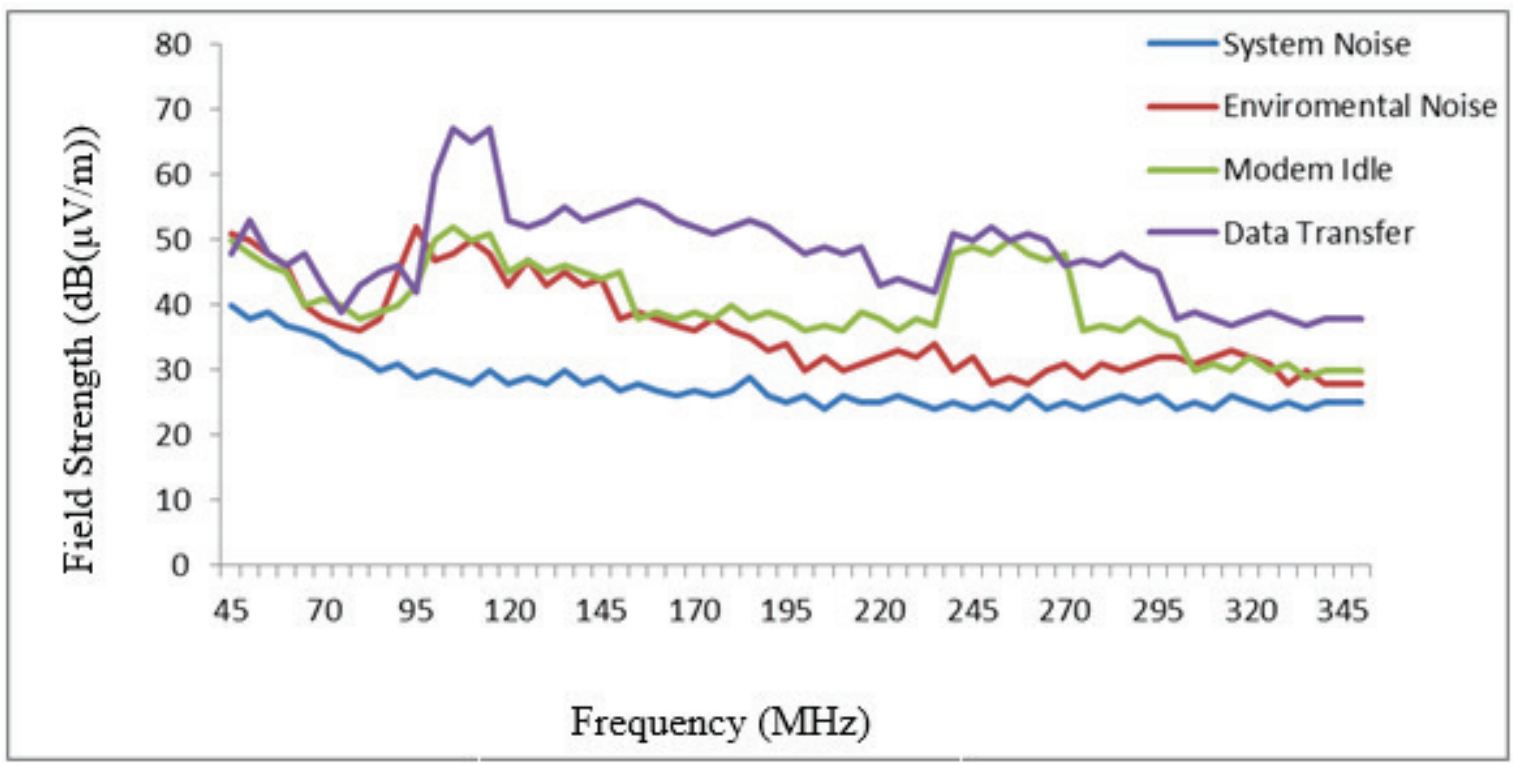

Figure 2: PLC Interference Field Strength with Vertical Polarization

\section{CONCLUSION}

There is much theoretical and practical evidence that PLC systems radiate wirelessly. The effect has been demonstrated for many frequency bands and is not limited to particular PLC systems. This study has analyzed the effect of PLC on radio communications equipment with the intent to reduce the interference radiation that emanates from a power line when used as radio communication. This study provides spectral measurements of the radiation from high-speed PLC modems in comparison with the signals from FM transmitters and DAB transmitter's band.

The results of the study showed that the radiations from PLC are comparable to the field-strengths for reception. The spectral measurements on high-speed PLC modems showed interference radiation occurring at frequencies up to $305 \mathrm{MHz}$, which includes the FM band and DAB band. The measurements also show that as the data rate of high speed modems increases, a corresponding increase in the interference radiation occurs at frequencies above $300 \mathrm{MHz}$.

The study has established that PLC systems on the specific situation turn power lines and household wiring into antennas though the strength of emissions. In addition, the effect of PLC on radio communication equipment is interference due to frequency jamming between the power line communication and the radio communication since the PLC has a higher frequency than most of the radio communication equipment.

\section{REFERENCES}

[1] Akarte, V., Punse, N. and Dhanorkar, A. (2014). Power line communication systems. International Journal of Innovative Research in Electrical, Electronics, Instrumentation and Control Engineering, 2(1): 709-713.

[2] Ashcroft, J., Daniels, D. J. and Hart, S. V. (2002). Guide for the selection of communication equipment for emergency first responders. National Institute of Justice, NIJ Guide 104-00; 1-64.

[3] Bhatia M, and Shamma, R. (2017). A review on power line communication. IEEE Communications Magazine, 4(9): 1-15.

[4] ITU-R SM. (2011). Impact of power line telecommunication systems on radio communication systems operating in the VHF and UHF bands above $80 \mathrm{MHz}$. SM Series Spectrum management, 1-72.

[5] Kouwen, A., Scanlon, M., Choo, K. R. AND Khac, N. A. (2010). Digital forensic investigation of two-way radio communication equipment and services. Forensics and Security Research Group, 1-26.

[6] Lampe, L., Newbury, J., Swart, T. and Ferreira, E. H. (2010). Power line communications. New York, NY: John Wiley \& Sons, 1-26

[7] Marcuzzi, F. and Tonello, A. M. (2018). Radio access network backhauling using power line communications. Broadband Communications Networks - Recent Advances and Lessons from Practice, 5975.

[8] Probir K. B. (2012). Introduction to radio communication. IEEE communication paper. 4(4): 78-86,

[9] Rapp, M., Dostert, K. and Gotz, M. (2004). Power line channel characteristics and their effect on communication system design, IEEE Communications Magazine, 42(4): 78-86.

[10] Rasi, H. and Gilan, M. S. (2016). New method for data communication over power-line career. International Journal of Advanced Biotechnology and Research (IJBR), 7(5): 993-1002. 
[11] Tang, S., Xie, Y., Tang, C. and Jinwenzhu, G. (2012). A comparative study of power line communication networks with and without Buffer. Int. Conference Information Networking (ICOIN), 4(2)

[12] Tonello, A. M., Versolatto, F., Bejar, B. and Zazo, S. (2012). “A Fitting Algorithm for Random Modeling the PLC Channel”, IEEE Transactions onPower Delivery 27 (3) (2012) 1477-1484.

[13] Tsuchiya, F. (2002). Measurements of harmful interference in the HF,UHF bands caused by extension of power line communication bandwidth. Int. Conference Inform. And Commun. Technologies: From Theory to Applications, 21: 1-14.

[14] UNDP. (2010). A guide to radio communication standards for emergency responders. Prepared Under United Nations Development Programme (UNDP) and the European Commission Humanitarian Office (ECHO) Through the Disaster Preparedness Programme (DIPECHO), Regional Initiative in Disaster Risk Reduction, 1-58.

[15] Varma, M. K., Jaffery, Z. A. and Ibraheem, K. (2019). "Broadband power line communication: the channel and noise analysis for a power line network", International Journal of Computer Networks \& Communications (IJCNC), 11(1), 81-92.

[16] Veronesi, D., Riva, R., Bisaglia, P., Osnato, F., Afkhamie, K., Nayagam, A., Rende, D. and Yonge, L. (2011). "Characterization of in-home MIMO power line channels", IEEE International Symposium on Power Line Communications and Its Applications, IEEE, 2011, pp. 42-47.

[17] Wikipedia (2019). Radio. https://en.wikipedia.org/w/index.php?title=Radio\&oldid=9.

[18] Yin, Y. (2017). "Application of PLC Control System and Communication Technology in Chemical Plant Equipment Renovation", Chemical Engineering Transactions, 62, 679-684. 